Meta

Journal des traducteurs

Translators' Journal

\title{
Pour une saine gestion de la documentation en terminologie et en traduction
}

\section{Robert Dubuc}

Volume 25, numéro 1, mars 1980

La documentation

URI : https://id.erudit.org/iderudit/002606ar

DOI : https://doi.org/10.7202/002606ar

Aller au sommaire du numéro

Éditeur(s)

Les Presses de l'Université de Montréal

ISSN

0026-0452 (imprimé)

1492-1421 (numérique)

Découvrir la revue

Citer cet article

Dubuc, R. (1980). Pour une saine gestion de la documentation en terminologie et en traduction. Meta, 25(1), 11-20. https://doi.org/10.7202/002606ar d'utilisation que vous pouvez consulter en ligne. 


\title{
Pour une saine gestion de la documentation en terminologie et en traduction
}

\author{
ROBERT DUBUC \\ chef adjoint du Service de linguistique, \\ Radio-Canada
}

L'exercice compétent de la profession de traducteur ou de terminologue ne repose plus aujourd'hui uniquement sur des connaissances linguistiques. Le traducteur et le terminologue doivent pouvoir compter, dans une très large mesure, sur une chose d'apparence aussi prosaïque que la documentation.

\section{DOCUMENTATION ET TRADUCTION}

On a abondamment parlé de la technicisation de la profession de traducteur. De fait, le professionnel de la traduction consacre la majeure partie de son temps et de ses énergies à des textes techniques ou paratechniques. Ces textes peuvent être de différents niveaux : scientifique, technique ou de vulgarisation. Mais dans un cas comme dans l'autre, tant pour le fond que pour la forme, le traducteur a besoin de documentation.

Ce besoin est d'autant plus impérieux que la spécialisation étroite du traducteur dans un domaine très circonscrit apparaît comme une vue de l'esprit. Dans la pratique, même le traducteur technique doit faire preuve de polyvalence. Ainsi dans une entreprise de médias où les textes techniques devraient normalement porter sur la radiotélévision, les traductions, au cours d'un mois type de travail, ont porté sur les thèmes suivants : politique de rémunération, descriptions de postes et monographies professionnelles (gestion); fabrication de l'huile de palme, recherche agricole aux États-Unis, culture de la betterave sucrière (agriculture). Donc rien sur les techniques et l'exploitation des médias, spécialités de la maison; mais des textes sur la gestion et l'agriculture. Si les textes de gestion ne nécessitaient guère de ressources documentaires nouvelles, par contre les textes en agriculture ont exigé un effort sérieux de documentation pour résoudre les problèmes de terminologie posés.

\section{FOND ET FORME}

Il ne s'agissait pas seulement pour le traducteur de se documenter pour comprendre ces textes, c'est-à-dire en saisir le fond. Il lui fallait savoir comment dire ces réalités, c'est-à-dire exprimer le message dans une forme idiomatique, qui donnerait au produit final la même allure qu'un original écrit sur le même sujet. La fonction documentaire doit donc porter autant sur le fond que sur la forme. 
Prenons l'exemple du texte sur la betterave à sucre.

Qu'est-ce que la documentation va apporter que le «dictionnaire bilingue » n'apporte pas? En gros, l'image d'une langue vivante en situation avec ses caractéristiques et son découpage particulier de la réalité.

\subsection{Richesse synonymique :}

Pour rendre sugar beet, on s'aperçoit que les textes parlent davantage de BETTERAVE SUCRIÈRE que de BETTERAVE À SUCRE.

Carbon dioxide correspond à GAZ CARBONIQUE dans la langue courante et à BIOXYDE DE CARBONE à un niveau plus technique.

CASSONADE dans la langue technique se dit du sucre de canne (brown sugar) ; le produit équivalent pour le sucre de betterave est VERGEOISE. Dans les textes de vulgarisation, cassonade est employé indifféremment pour la canne et la betterave.

Pour rendre sugar factory (terme générique), on emploie en français un spécifique de produit BETTERAVERIE ou de fonction RAFFINERIE. De même l'idée de seed est rendue tantôt par le terme abstrait SEMENCE tantôt par le terme concret GRAINE.

2.0 Termes propres à la technique :

2.1 De niveau conceptuel

\begin{tabular}{|c|c|}
\hline (culture) & $\begin{array}{l}\text { seed bed }=\text { lit de semence } \\
\text { beet leaf }=\text { fanne } \\
\text { common red beet }=\text { betterave potagère } \\
\text { close planting }=\text { semis dense } \\
\text { space planting }=\text { semis de précision } \\
\text { sugar grower }=\text { betteravier }\end{array}$ \\
\hline (récolte) & $\begin{array}{l}\text { topping }=\text { décolletage } \\
\text { topper }=\text { décolleteuse (machine) } \\
\text { harvester = ramasseuse (machine) } \\
\text { receiving station = poste de réception }\end{array}$ \\
\hline (traitement) & $\begin{array}{l}\text { scale }=\text { poste de pesée } \\
\text { beet cutter = coupe-racines } \\
\text { centrifuge = centrifugeuse (machine) } \\
\text { sugar-maker = sucrier (professionnel) } \\
\text { sugar-boiler = raffineur (professionnel) } \\
\text { carbonation = carbonatation } \\
\text { sweet juice = jus sucré } \\
\text { raw juice = jus de diffusion } \\
\text { clear juice = jus clair } \\
\text { non-sugar substances }=\text { les non-sucres } \\
\text { diffusion cell }=\text { trommel de diffusion } \\
\text { proof stick = pipette } \\
\text { muddy sediments }=\text { boues } \\
\text { fillmass }=\text { masse cuite }\end{array}$ \\
\hline
\end{tabular}


2.2 De niveau fonctionnel

(culture) to thin the beets $=$ démarier to cultivate soil $=$ passer le champ au cultivateur

(récolte) to top $=$ décolleter

(traitement) to seed a liquid for crystallization = amorcer la cristallisation to granulate $=$ grainer

3.0 Découpage différent de la réalité :

Salad-maker or beet cutter : l'anglais emploie tantôt le terme familier salad-maker tantôt le terme technique beet cutter. Le français ne semble connaître que le terme technique COUPE-RACINES.

Pour désigner le sucre qui colle au fond du récipient par suite d'une évaporation trop intense, l'anglais emploie le substantif scorching, le français, le verbe intransitif ATTACHER. Exemple : Pour éviter que le sucre n'attache.

Pour nommer l'opération par laquelle les botanistes mettent au point de nouvelles variétés de plantes, l'anglais emploie le substantif abstrait hybridation, le français, le terme concret : CROISEMENT(S).

L'opération qui consiste à débarrasser le jus de diffusion de son excédent d'eau se nomme en anglais evaporation et en français CONCENTRATION, ce qui constitue un joli chassé-croisé de la cause à l'effet.

Cette longue suite d'exemples ne vise qu'à montrer la nécessité de la documentation pour pouvoir donner au texte traduit la couleur, le teint, la vigueur et la crédibilité d'un original.

\section{LE RISQUE DE POLLUTION}

Traduire, ce n'est nullement calquer une langue de départ dans une langue d'arrivée, mais bien exprimer dans une langue d'arrivée, selon les ressources de cette langue, un texte pensé, conçu et exprimé dans une autre langue. La clé de voûte de cette opération apparaît tenir dans une bonne documentation.

Le traducteur qui invente sa terminologie avec les moyens du bord, sans prendre la peine de se documenter, multiplie les conventions de communication en mettant en circulation des terminologies parallèles. Il en résulte des difficultés accrues de communication. Paraphrasant Bernard Shaw, on pourrait dire que la traduction non documentée sépare les locuteurs d'une même langue.

La façon de remédier à cette pollution consiste à mettre la documentation à la disposition du traducteur. La solution est plus facile à exprimer qu'à mettre en œuvre. Pourtant, il existe de nombreuses possibilités. 


\section{LES RESSOURCES DE L'AUTOMATISATION}

Dans les bureaux de traduction et même dans les centres de terminologie, on a fort peu exploité les ressources des services de documentation automatique. Ces services ont été jusqu'ici orientés vers les bibliothèques plutôt que mis à la portée des utilisateurs directs.

D'autres personnes traiteront sans doute de cet aspect dynamique de la documentation. Qu'il suffise pour l'instant de mentionner quelques-uns de ces systèmes dont on pourrait déjà tirer un certain profit : le fichier bibliographique automatique de l'Office de la langue française du Québec (Terminoq II), le système DATUM (information juridique), le système BADADUQ de l'Université du Québec, le système INFORMATECH-SABINE, qui touche à un très grand nombre de domaines techniques et le système CANOLE, fichier bibliographique central des bibliothèques canadiennes.

L'idée des banques de terminologie lancée d'une façon fonctionnelle au début des années 70 avait fait peut-être naître trop d'espérances pour ne pas engendrer de déceptions. Il semble que dans l'élaboration de ces vastes projets, on ait manqué de modestie et qu'on ait négligé de définir des objectifs de service précis à un système de documentation automatique spécialisé qui s'engorge apparemment dans sa complexité.

Alors que la conception d'un dictionnaire automatique à orientation de terminologie bilingue semblait un projet réalisable à plus ou moins court terme, la mise en place d'un système capable de répondre aux besoins terminologiques de toutes les catégories d'usagers est beaucoup plus ambitieuse et plus complexe à mettre en œuvre.

Les banques de terminologie au Canada visent à offrir un service soit ponctuel, soit thématique, pour aider l'usager à résoudre ses problèmes de terminologie. La formuie du fichier automatisé à la base de ces systèmes est vraiment très alléchante; elle permet l'inversion automatique des fiches, le regroupement par domaines et sous-domaines, les combinaisons analogiques et l'accessibilité instantanêe à l'information. De plus, l'accès peut être autant ponctuel que thématique et les clés d'accès à une même fiche peuvent être multiples, ce qui assure une utilisation maximale de la matière emmagasinée.

Le jour n'est peut-être plus très loin où chaque traducteur travaillera avec son écran cathodique, branché directement sur une ou plusieurs banques de terminologie. À ce moment, les problèmes de documentation passeront $d u$ traducteur au terminologue. Parce que l'adage informatique « garbage in, garbage out $»$ se vérifie principalement dans une banque de terminologie.

Si l'information terminologique mise en mémoire ne repose pas sur une documentation solide, on retombe de Charybde en Scylla. Il importe donc qu'à un moment donné de l'opération terminologie-traduction intervienne l'opération documentation, reposant sur un service de documentation. 


\section{EXPLOITATION D'UN SERVICE DE DOCUMENTATION}

La qualité de la documentation étant absolument nécessaire à la qualité des traductions techniques, il appert que le service de documentation doit répondre de façon compétente aux besoins des traducteurs, mais à l'intérieur d'une fourchette de coûts raisonnables. Si l'accès à une bonne documentation ralentit trop le débit du travail des traducteurs, il y a de gros risques que l'employeur mette la hache dans le service de documentation sans autre forme de procès. Le service de documentation doit travailler vite et bien. Cette conciliation, cela va de soi, n'est pas toujours facile. Mais il faut se rappeler que le temps de recherche coûte cher et que les clients sont pressés. Ce sont des considérations dont on ne peut faire abstraction si l'on veut fonctionner efficacement.

Le problème essentiel du service de documentation en traduction et en terminologie est donc de fournir rapidement des renseignements documentaires de qualité.

\section{STRUCTURE}

Le service de documentation doit se composer de deux volets : un secteur d'information brute, textes généraux et spécialisés sur les sujets traités par l'entreprise, un secteur d'information raffinée où des terminologues ont adapté aux besoins précis du traducteur l'information brute.

Dans le secteur d'information brute doivent se retrouver la documentation grammaticale, les encyclopédies et dictionnaires de langue, les dictionnaires bilingues ou multilingues, les ouvrages et traités reliés aux domaines d'activité de l'entreprise.

Dans le secteur d'information raffinée se retrouvent essentiellement le fichier terminologique (fiches terminologiques établies par les traducteurs et terminologues) et le fichier-sujets établi par le documentaliste avec l'aide des terminologues.

Pour arriver à une efficacité satisfaisante, il faut définir, pour le secteur brut, des circuits de recherche selon les domaines où les ouvrages consultés vont par ordre croissant de difficulté de consultation. Par exemple, pour une difficulté grammaticale, on ira du dictionnaire de difficultés (de consultation facile) au Grevisse (plus compliqué) jusqu'aux ouvrages spécialisés de linguistique (ordinairement très compliqués).

Pour la terminologie, d'abord dictionnaires bilingues ou multilingues, puis dictionnaires encyclopédiques, encyclopédies, dictionnaires spécialisés et enfin ouvrages, traités, monographies (de consultation lente et difficile).

L'efficacité du secteur brut exige aussi un mode fonctionnel de classement. Au risque de s'attirer les foudres de tous les bibliothécaires réunis, il faut quand même affirmer que les classifications classiques en usage dans les bibliothèques conviennent mal aux petits services de documentation. C'est comme faire tourner un disque en utilisant un amplificateur de 100 watts pour une pièce de $3 \mathrm{~m}$ sur $3 \mathrm{~m}$. C'est trop \& puissant 》 pour les besoins. 
L'expérience semble attester que le système qui donne le meilleur résultat est celui de la roue de spécialité. Pour chaque domaine où l'entreprise cuvre prioritairement, on établit un mode de classement fondé sur une espèce de graphique à secteur, dont le moyeu est la spécialité. La spécialité se divise selon les besoins en cinq ou six secteurs, qui constituent les rubriques de classement. Sous chaque rubrique, on pourra dresser la liste des descripteurs pertinents par ordre alphabétique (voir fig. I). Il faut se garder de la tentation de raffiner à l'excès ses rubriques de classement. Dans un service de documentation axé sur la terminologie et la traduction, chaque rubrique doit être un pôle de regroupement de l'information qui permet de la repérer facilement. Il ne s'agit pas d'opérer un classement rationnel des notions pour un secteur d'activité donné.

L'avantage supplémentaire de la roue, c'est qu'elle peut servir aussi de mini-répertoire de domaines aux terminologues. Il y a donc correspondance des classements entre la matière brute et la matière raffinée.

FIGURE I

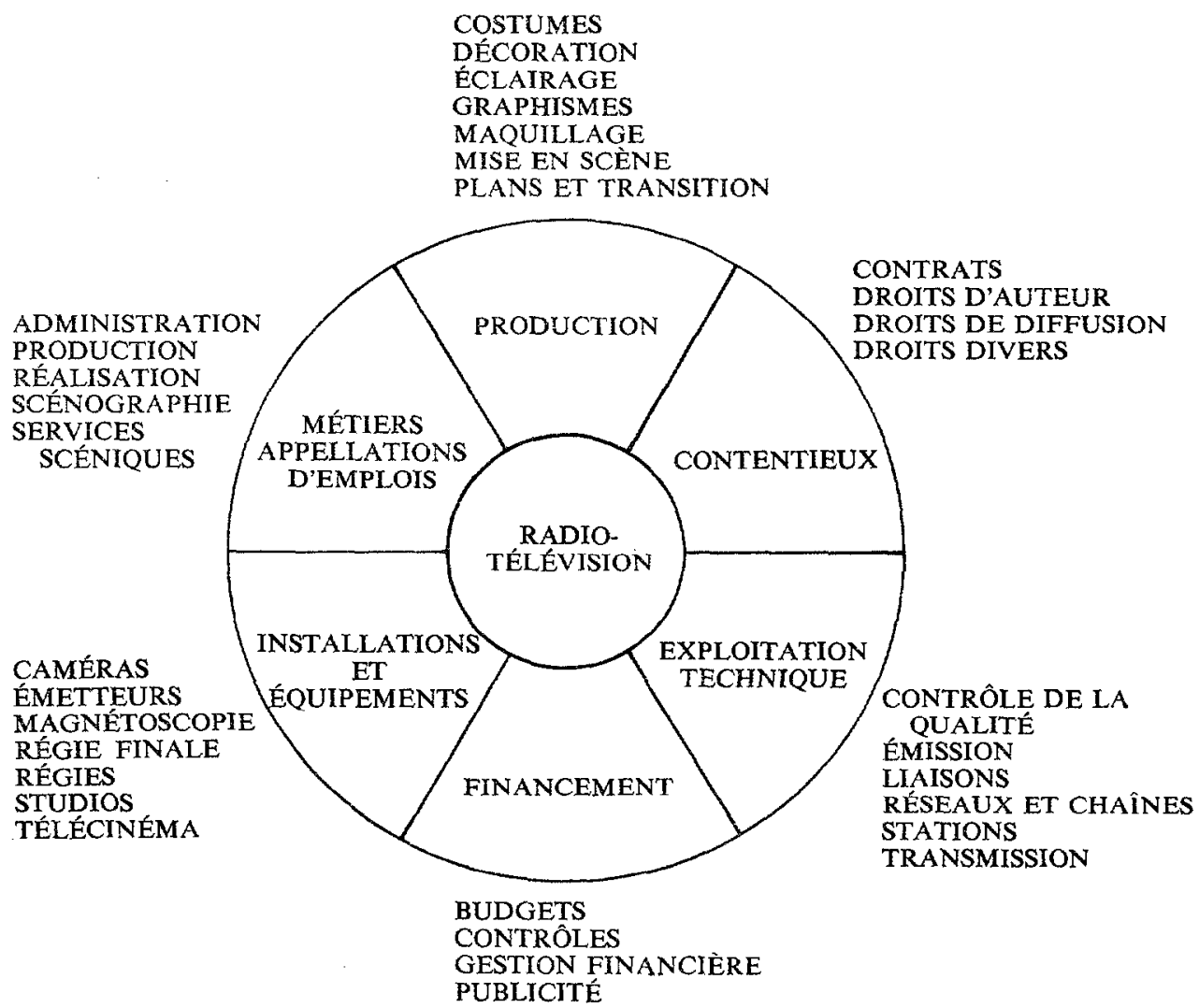




\section{LE SECTEUR RAFFINE}

Pourquoi le service de documentation ne peut-il se contenter d'offrir de la documentation brute à ses traducteurs? C'est une pure question d'économie à long terme. Le salaire horaire moyen du traducteur doit bien se situer aux environs de $\$ 10$ pour le traducteur d'entreprise (et à $\$ 20$ pour le pigiste). Chaque heure passée dans la documentation brute par le traducteur coûte cher. Tandis que l'information trouvée au fichier terminologique est rapidement exploitable, donc extrêmement rentable. L'établissement d'un fichier terminologique, en plus d'accélérer le débit de traduction, produit des retombées intéressantes pour uniformiser la terminologie en usage, éviter la duplication des recherches et garantir la validité de l'information.

Le fichier terminologique d'un service de documentation doit être central, universel et vivant. Il doit être central parce qu'il doit être la première source consultée, et par conséquent, d'accès facile à tous les utilisateurs. Il doit être universel. Toutes les fiches doivent y être versées dans l'ordre alphabétique le plus strict et le plus bête, de façon que n'importe qui puisse y classer des fiches sans risque d'erreur. Les classements raisonnés pour la consultation courante sont à proscrire parce qu'ils compliquent le repérage de l'information. En faisant trop appel au raisonnement, ils introduisent dans le classement une dimension subjective qui joue au détriment de l'efficacité de la consultation.

Le fichier doit être vivant, c'est-à-dire qu'on doit y verser constamment des fiches nouvelles qui consignent les résultats des recherches ponctuelles et thématiques des traducteurs et des terminologues ou qui sont établies par suite du dépouillement des revues spécialisées. Bien que les fiches versées au fichier doivent être documentées, c'est-à-dire tirées d'une source valable, elles n'ont pas à être nécessairement complètes ou définitives. Un fichier vivant est au diapason de la langue vivante, comme dirait $M$. Lapalisse. Ce n'est pas le repaire de l'orthodoxie académique.

En somme on pourrait se représenter le service de documentation sous la forme d'une chaîne de production (fig. II). Les documents (encyclopédies, dictionnaires, manuels, textes divers) sont la matière première de la recherche. Cette recherche est facilitée par l'établissement d'un fichier-sujets, suite au dépouillement sommaire des documents. $\AA$ l'aide du fichier-sujets, on peut constituer un dossier documentaire à l'intention du client, qui devra, le cas échéant, tirer de ce dossier les renseignements dont il aura besoin pour traduire son texte.

Pour arriver à la documentation raffinée, ou ponctualisée si l'on peut dire, le terminologue, à l'aide des dossiers documentaires, procède au dépouillement des textes pour en tirer des fiches qui seront versées au fichier central, directement consultable par l'usager.

De fait, en théorie du moins, la constitution d'un dossier documentaire devrait donner lieu à la création de fiches; en pratique, les contraintes de temps et d'organisation ne le permettent pas toujours. 
META XXV, 1

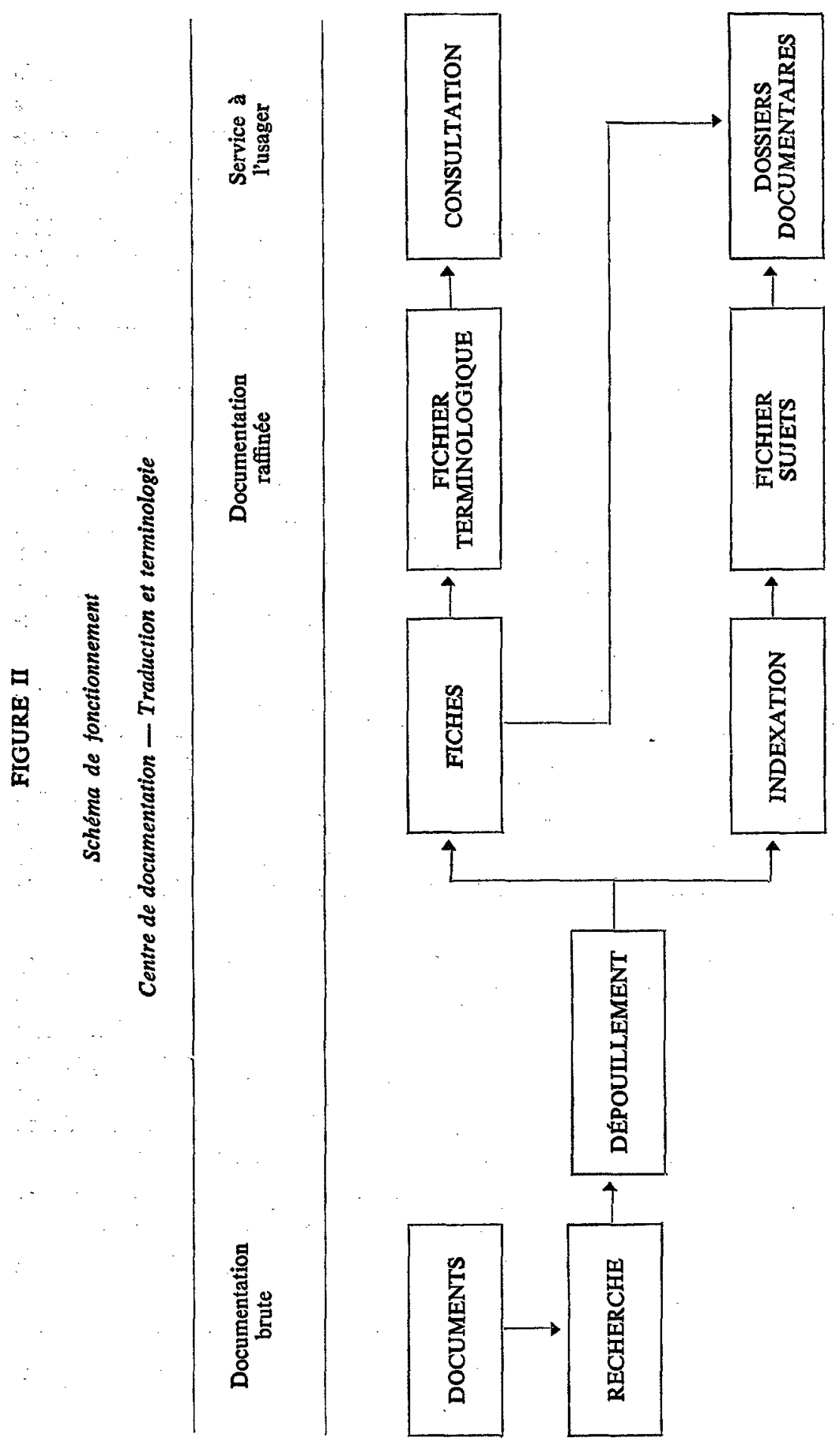


L'activité d'un service de documentation orientée vers la traduction et la terminologie, met en relief la nécessité d'introduire dans le paysage un nouveau personnage : le documentaliste spécialisé en traduction-terminologie.

Évidemment, pour justifier la présence d'un documentaliste, le service de documentation doit avoir une certaine ampleur et desservir un nombre d'usagers tel qu'il peut rentabiliser sa fonction. C'est à chaque entreprise d'évaluer sa situation et de juger de la nécessité d'un documentaliste. Dans certains cas, le documentaliste apparaîtra comme un luxe, mais un luxe d'une très grande utilité !

La présence d'un documentaliste vise essentiellement à permettre l'utilisation maximale des documents accumulés. L'expérience nous enseigne qu'une documentation non gérée par quelqu'un de compétent est en général exploitée au dixième de ses virtualités. Le dépouillement sommaire des documents ne se fait pas; il n'y a pas de fichier-sujets; les documents épars sont complètement négligés; ce ne sont de fait que les documents de consultation facile qui servent. On ne tire pas parti, en général, des dossiers documentaires constitués pour une recherche donnée. Il y a donc souvent duplication des recherches et manque d'uniformité dans les résultats.

\section{PROFIL DU DOCUMENTALISTE}

Pour répondre aux besoins de la terminologie et de la traduction, le documentaliste doit certes avoir une formation professionnelle acquise soit au CEGEP (techniques de documentation) soit sur le tas (travail en bibliothèque, dans les centres de documentation, etc.). Ce n'est pas le diplôme qui compte, mais la compétence.

La compétence d'un documentaliste dans un domaine d'activité aussi spécialisé tient beaucoup à sa capacité de concevoir les problèmes dans une optique qui transcende la technique acquise. Il doit bien se garder de prendre ses techniques pour une fin, mais plutôt les considérer comme des moyens de résoudre les problèmes qui lui sont posés.

Le documentaliste doit avoir une grande ouverture d'esprit, une inaltérable curiosité, de l'imagination et de l'intuition pour tracer des cheminements documentaires courts et efficaces. Mais la qualité qu'il faut en exiger par-dessus tout c'est la connaissance des problèmes de terminologie et de traduction et des types particuliers de solutions qu'ils appellent. Cette sensibilité, si elle n'est pas déjà acquise, doit l'être dans les trois mois du stage, sinon on s'expose à de perpétuelles frustrations de part et d'autre. Le documentaliste doit comprendre que son rôle dans ce contexte est de permettre à ses clients de travailler plus vite et mieux. Il faut donc, pour reprendre une formule usée, qu'il soit plus «trouveur » que «chercheur». Il ne faudrait pas conclure de l'emploi du masculin fonctionnel que ce poste ne peut être rempli par une femme. Le sexe n'a rien à voir avec les qualités essentielles du documentaliste.

Si l'on veut être brutal, disons que pour justifier sa présence, le documentaliste doit permettre aux traducteurs et terminologues d'éviter de 1500 à 2000 heures de recherche par année. Au-delà de ce seuil, sa présence devient rentable. 
En ce sens, sa capacité d'établir des liens avec d'autres services similaires pourra se révéler d'une grande rentabilité. Il lui appartiendra aussi de dresser un fichier d'experts auxquels on peut faire appel selon les besoins. En retour, il devra rendre des services à l'extérieur. Le bon documentaliste sera très peu «fonctionnarisé » dans sa façon de concevoir son travail.

\section{CONCLUSION}

En raison de la polyvalence exigée des traducteurs et des terminologues, il faut à ceux-ci pour exercer leur métier de façon compétente l'accès à une documentation qui leur permette non seulement de connaître les réalités du domaine où ils cuvrent, mais aussi d'exprimer ces réalités d'une façon idiomatique.

Pour atteindre cet objectif, les services de traduction et de terminologie doivent pouvoir compter sur des ressources documentaires externes : service de documentation automatisée, banques de terminologie, mais aussi sur des ressources internes : constitution d'une documentation directement axée sur les besoins d'expression de l'entreprise. L'exploitation efficace et la gestion de ce fonds documentaire exigent la présence d'une personne compétente - documentaliste spécialisé - qui assure le rendement maximal du fonds documentaire, et partant celui des traducteurs et des terminologues. C.Q.F.D. 